\title{
Interactions:
}

\section{Having an Impact}

Near the end of this issue is a piece by Keith Instone and Fred Sampson that marks the passing of Randy Pausch. Jon had the good fortune of taking one of Randy's classes during his junior year at Carnegie Mellon, and while individual lectures may now be forgotten, the dramatic influence and impression of Randy's passion is forever lasting. Randy's contributions to design, entertainment, and education are phenomenal, and the personal impact he had on students and colleagues, and eventually on millions around the globe, indicates the substantive and often unpredictable influence that our ideas, creations, and pursuits can have on the world we leave behind.

This notion of influence-of leaving a mark-is one that is reflected in different ways in contributions by several authors, including Ben Bederson, who, via the International Children's Digital Library (ICDL), is returning pleasure reading to children in Mongolia and, for children elsewhere, increasing access to books from other cultures. In another article, Rich Ling describes some of the extraordinary impact mobile phones have had on our lives. But the impact of mobile phones has not been universally positive. Neither has that of email: Karen Renaud and colleagues provide an analysis of its seriously negative effects. As Brian Romanko suggests in his review of one of Ling's books, while certain forms of technology-supported communication have become indispensible, they have also become a sociological pain.

Ben and his colleagues describe how they are on the lookout for and are designing to prevent any sort of unwanted influence of the ICDL, such as the loss of meaningful engagement with books when they are accessed via the Web. Other authors offer guidance to increase the chances of the positive impact of others' work. For example, Liz Sanders presents an evolving map of design research that can help ensure that the newest approaches are considered for use along with the old. Christine Satchell provides explanation and examples of how cultural theory should influence research and design. Mike Kuniavsky discusses important guidelines for those engaged in the design of the ubiquitous computing user experience. As Don Norman puts it, "As we move from the world of stand-alone objects to social structures, complex, intelligent products, and a heavy dominance of services, then new principles are needed."

And according to Nathan Shedroff, arguably the father of "experience design," businesses need to follow new organizational and management principles as well so that they can create the proper context and culture to enable the right process for design and innovation. Nathan discusses how this relates to the focus of his upcoming booksustainability. Bill Tomlinson describes how corporations (and individuals) can continue their natural pursuit of conspicuous consumption, but in ways that are beneficial to the environment.

However, before you get to all of that, the issue opens with our cover story by Sus Lundgren, who explores the interaction paradigms embedded in games and the moral or ethical issues they raise. The design strategies Sus advocates have furtherreaching impact than one might initially think, as they are applicable to any experience or environment in which creative and abstract rule definition must occur. Games are one of these settings; business, education, and politics are others.

This issue is all about having an impact. The ability to have an impact via changing behavior is at the heart of interaction design; it's at the heart of the late Randy Pausch's career, and it's at the core of interactions.

$$
\text { -Richard Anderson and Jon Kolko }
$$
eic@interactions.acm.org

\footnotetext{
DOI: $10.1145 / 1409040.1409041$

(C) 2008 ACM 1072-5220/08/1100 $\$ 5.00$
}

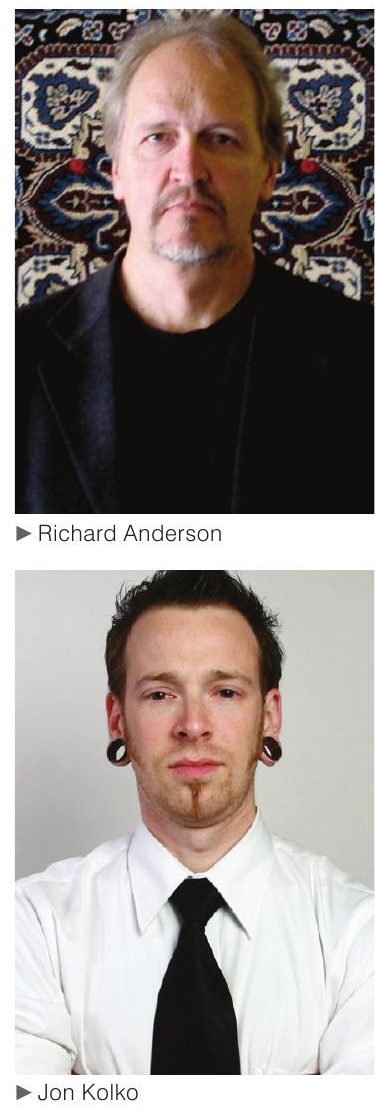

Jon Kolko 